\title{
Giovanni Giacometti: On the Occasion of His 85th Birthday
}

\author{
Marilena Di Valentin • Donatella Carbonera
}

Published online: 7 March 2015

(C) Springer-Verlag Wien 2015

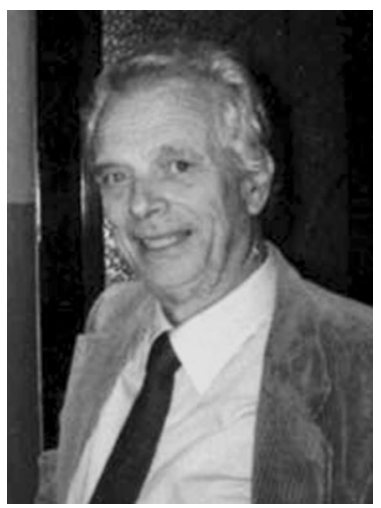

On the 6th of September 2014, we celebrated the 85th birthday of Giovanni Giacometti. To mark this special date, Prof. Kev M. Salikhov decided to prepare a special issue of Applied Magnetic Resonance dedicated to Giovanni. As former students and close collaborators, we were asked to participate in the role of guest editors and we were very pleased to invite his colleagues and friends to contribute to this issue. We were delighted that they did not hesitate to accept as a proof of the high esteem for his contribution to the field of EPR spectroscopy.

Giovanni Giacometti graduated from the Chemistry Department of the University of Padova in 1951 and received the degree of "Libera Docenza" (Ph.D.) in Physical Chemistry in 1955 after working in the laboratories of Linus Pauling in Pasadena and Robert Mulliken in Chicago. As a post-doc at the National Research Council in Ottawa (Canada), he was introduced to EPR spectroscopy, combined to

M. Di Valentin $(\bowtie) \cdot$ D. Carbonera

Department of Chemical Sciences, University of Padova, Via F. Marzolo 1, Padua, Italy e-mail: marilena.divalentin@unipd.it 
photochemistry and radical chemistry. He has been Professor of Theoretical and Physical Chemistry at the University of Padova since 1960, where he is now Professor Emeritus. From 1977 to 1981, he was Dean of the Faculty of Sciences and from 1970 to 2000 he was the Director of the National Research Council Center of Studies for Radical and Excited Molecular States. He was granted the Italian gold medal for Science and Culture in 1970 and the "G.B. Bonino" gold medal from the Italian Chemical Society in 2001. He is a member of the prestigious "Accademia Nazionale dei Lincei", the "Istituto Veneto di Scienze Lettere ed Arti" and a foreign member of the "Societé Royale des Sciences" de Liège. In 2006 he was nominated "Honorary Professor" at the University of Sichuan, Chengdu, China.

Combining his deep interest in theoretical chemistry and spectroscopy, Giovanni Giacometti founded the first research group in Electron Spin Resonance in Italy. Starting from the Giacometti-Nordio-Pavan modification of the McConnell's relation between proton hyperfine splittings and spin densities (Theoret. Chim. Acta 1963, 1, pp. 404-409), the group gave a number of relevant and wide-ranging contributions to the field, both on theoretical and experimental problems related to the electronic properties of organic radicals, photoexcited charge transfer pairs and triplet states. The goal of interdisciplinarity has always accompanied his research projects from the beginning of his long scientific career. His great scientific versatility and his deep conviction that diverse aspects of biology should be based on the rules of quantum mechanics, led him to biophysics applying the advanced EPR techniques, in particular Optically Detected Magnetic Resonance, to the investigation of the molecular mechanisms of photosynthesis focusing his work on photoexcited paramagnetic species in natural and biomimetic complexes. Photosynthesis became his favorite topic since he could finally combine all his scientific interests and collaborate with his brother Giorgio Giacometti at the Department of Biology. His enthusiasm and open-minded approach has also favored internationalization, establishing solid collaborations with groups around the world, which are still maintained. Several of these collaborations evolved into enduring friendships.

Giovanni Giacometti has been not only a man of science but he has also been involved in civil issues, actively contributing to the strengthening of the democratic institutions in Italy in the most difficult political periods. An example was his firm position as Dean of the Faculty at the time, when Italy was enduring a bitter struggle against terrorism. He has always shared this passion with his colleagues and every scientific discussion would alternate with brief interludes on the Italian policy.

We are indebted to Giovanni for the enthusiasm and love for science that he was able to instill in both of us and in all the students of the biophysical group in Padova.

We heartily thank all the contributors and we are grateful to Prof. Kev Salikhov and Dr. Laila Mosina for their support and encouragement in all phases of the preparation of this special issue.

We wish Giovanni good health in the years to come. 